\title{
The Effect of de-Sitter Like Background on Increasing the Zero Point Budget of Dark Energy
}

\author{
Haidar Sheikhahmadi, ${ }^{1,2}$ Ali Aghamohammadi, ${ }^{3}$ and Khaled Saaidi ${ }^{2}$ \\ ${ }^{1}$ Institute for Advanced Studies in Basic Sciences (IASBS), Gava Zang, Zanjan 45137-66731, Iran \\ ${ }^{2}$ Department of Physics, Faculty of Science, University of Kurdistan, Pasdaran Street, Sanandaj 66177-15175, Iran \\ ${ }^{3}$ Islamic Azad University, Sanandaj Branch, Pasdaran Street, P.O. Box 618, Sanandaj, Iran
}

Correspondence should be addressed to Haidar Sheikhahmadi; h.sh.ahmadi@gmail.com

Received 9 April 2016; Accepted 18 May 2016

Academic Editor: S. Habib Mazharimousavi

Copyright (C) 2016 Haidar Sheikhahmadi et al. This is an open access article distributed under the Creative Commons Attribution License, which permits unrestricted use, distribution, and reproduction in any medium, provided the original work is properly cited. The publication of this article was funded by SCOAP ${ }^{3}$.

\begin{abstract}
During this work, using subtraction renormalization mechanism, zero point quantum fluctuations for bosonic scalar fields in a de-Sitter like background are investigated. By virtue of the observed value for spectral index, $n_{s}(k)$, for massive scalar field the best value for the first slow roll parameter, $\epsilon$, is achieved. In addition, the energy density of vacuum quantum fluctuations for massless scalar field is obtained. The effects of these fluctuations on other components of the universe are studied. By solving the conservation equation, for some different examples, the energy density for different components of the universe is obtained. In the case which all components of the universe are in an interaction, the different dissipation functions, $\widetilde{Q}_{i}$, are considered. The time evolution of $\rho_{\mathrm{DE}}(z) / \rho_{\text {cri }}(z)$ shows that $\widetilde{Q}=3 \gamma H(t) \rho_{m}$ has the best agreement in comparison to observational data including CMB, BAO, and SNeIa data set.
\end{abstract}

\section{Introduction}

For a de-Sitter like background, to investigate the effects of the quantum fluctuations on the energy budget of the universe both massive and massless scalar fields are considered. Because of the appearance of the first slow roll parameter, the relation between power spectral index and slow roll parameters leads to a way to connect theoretical results with observations. In fact one can estimate the best value of the first slow roll parameter based on observations, for instance, Planck 2013 [1]. From this perspective, for zero point quantum fluctuations both energy density and pressure are calculated. Interestingly it is observed that the contribution of zero point energy is increased against the results for the normal de-Sitter ones $[2,3]$. The importance of this note is that whereas the zero point contribution of dark energy potentially is detectable, therefore the possibility of dark energy detection is increased. Also it should be stressed, due to time dependency of Hubble parameter which appeared in energy density of zero point quantum fluctuations, energy can transfer between different components of the universe. By considering this concept one can propose different manners to investigate interaction between zero point fluctuations and other sectors. In a general case where all components are in an interaction, three different dissipation functions $\widetilde{Q}$ are considered. For this special case time evolution of $\rho_{\mathrm{DE}}(z) / \rho_{\mathrm{cri}}(z)$ shows that $\widetilde{Q}=3 \gamma H(t) \rho_{m}$ is in best agreement in comparison to observations including $\mathrm{CMB}, \mathrm{BAO}$, and SNeIa data set. Furthermore, energy density of matter, $\rho_{m}$, has some deviations in comparison to the result of ordinary de-Sitter one. In fact in $\rho_{m}$, beside $a^{-3}$, some extra terms appeared, where these new terms could be interpreted as new source for cold dark matter risen from interaction between matter and quantum fluctuations. In fact as it was discussed in [3] zero point quantum fluctuations could be considered as subdark energy, and these extra terms in question can be proposed as subdark matter. The scheme of this paper is as follows.

In Section 2 the general framework of this work including the mathematical calculations is discussed. In Section 3 the 
cosmological role for zero point energy density is investigated, and the results of this work are compared with previous works. In Section 4 to estimate the amount of subdark energy and also subdark matter, the bounds which are risen from time evolution of dark energy are considered. And at last, we have conclusion.

\section{Massive Scalar Field and Slow Roll Parameters}

To study the effect of zero point quantum fluctuations let us consider a real minimally coupled bosonic scalar field $\Phi$ in a semiclassical general relativity mechanism [4]. In such scenario the geometry is not quantized but the energy momentum tensor related to the scalar field is calculated by means of the vacuum expectation value concept. To begin we consider the action

$$
S=S_{m}+\int d^{4} x \frac{\sqrt{-g}}{\kappa^{2}}\left(R-2 \Lambda+\kappa^{2} \mathscr{L}_{\Phi}\right),
$$

where $S_{m}$ is the matter action, $g$ is the determinant of the metric, $R$ is the Ricci scalar, $\Lambda$ is the Einstein's cosmological constant, and $\mathscr{L}_{\Phi}$ is defined as

$$
\mathscr{L}_{\Phi}=-\frac{1}{2} g^{\mu \nu}\left(\nabla_{\mu} \Phi \nabla_{\nu} \Phi\right)-V(\Phi),
$$

where $V(\Phi)$ is the potential of the model. Variation of the action (1) with respect to metric yields

$$
R_{\mu \nu}-\frac{1}{2} g_{\mu \nu} R+g_{\mu \nu} \Lambda=\kappa^{2}\left(T_{\mu \nu}^{M}+\left\langle 0\left|T_{\mu \nu}^{\Phi}\right| 0\right\rangle_{\text {ren }}\right),
$$

where $T_{\mu \nu}^{M}=(-2 / \sqrt{-g}) \times\left(\delta S_{m} / \delta g^{\mu \nu}\right)$ and

$$
T_{\mu \nu}^{\Phi}=\partial_{\mu} \Phi \partial_{\nu} \Phi-\frac{1}{2} g_{\mu \nu}\left(\partial_{\alpha} \Phi \partial^{\alpha} \Phi+2 V(\Phi)\right)
$$

Using the above equation, the 00 and $i i$ components of energy-momentum tensor read

$$
\begin{gathered}
T_{0}^{\Phi 0}=-\frac{1}{2}\left[\dot{\Phi}^{2}+\frac{\Phi_{, i}^{2}}{a^{2}(t)}+2 V(\Phi)\right], \\
\frac{1}{3} T_{i}^{\Phi i}=\frac{1}{2}\left[\dot{\Phi}^{2}-\frac{1}{3} \frac{\Phi_{, i}^{2}}{a^{2}(t)}-2 V(\Phi)\right] .
\end{gathered}
$$

It is also obvious that variation (1) with respect to $\Phi$ yields

$$
\square \Phi-V_{, \Phi}(\Phi)=0,
$$

where $\square$ is d'Alembert operator. As it is mentioned above in semiclassical approach one can quantize the scalar field and therefore the vacuum expectation value of energy density and pressure could be obtained. For this purpose, the quantized scalar field is defined as

$$
\begin{aligned}
& \Phi(t, \mathbf{x}) \\
& \quad=\int \frac{d^{3} k}{(2 \pi)^{3} \sqrt{2 k}}\left[a_{\mathbf{k}} \phi(t) e^{-j \mathbf{k} \cdot \mathbf{x}}+a_{\mathbf{k}}^{\dagger} \phi^{*}(t) e^{j \mathbf{k} \cdot \mathbf{x}}\right],
\end{aligned}
$$

where $\phi(t)$ is a function which should be determined, $a_{\mathbf{k}}\left(a_{\mathbf{k}}^{\dagger}\right)$ is annihilation (creation) operators and $\sqrt{-1}=j[5,6]$. Now introducing (7) to (6), it expresses

$$
\ddot{\phi}+3 H(t) \dot{\phi}+\frac{k^{2}}{a^{2}} \phi+V_{, \phi}=0,
$$

where $H=\dot{a} / a$, and $a(t)$ is the scale factor of the universe, overdot denotes derivation with respect to the cosmic time, and the $k^{2} \phi / a^{2}$ term appeared due to scalar field's spatial dependency. For more convenience one can use conformal time $\eta=\int d t / a(t)$, and therefore (8) could be rewritten as

$$
\phi^{\prime \prime}+2 \mathscr{H} \phi^{\prime}+k^{2} \phi+a^{2} \frac{V^{\prime}(\phi)}{\phi^{\prime}}=0,
$$

in which overprime indicates derivation with respect to $\eta$, and $\mathscr{H}=a^{\prime} / a=\dot{a}$ is the conformal, comoving, Hubble parameter. Now, by defining $\chi:=\phi / a$, using a power law potential, $V(\phi)=m^{2} \phi^{2} / 2$, relation (9) reads

$$
\chi^{\prime \prime}+\left(k^{2}+m^{2} a^{2}-\frac{a^{\prime \prime}}{a}\right) \chi=0 .
$$

It should be stressed for de-Sitter like background in question that the scale factor is taken as $a(t)=t^{1 / \epsilon}$, where $\epsilon=$ $-\dot{H} / H^{2} \ll 1$ is the first slow roll parameter [7, 8]. Using definitions of scale factor (i.e., $a(\eta) \simeq-(1+\epsilon) / H \eta$ ) and second slow roll parameter, $\tau=m^{2} / 3 H^{2}$, one can attain

$$
-m^{2} a^{2}+\frac{a^{\prime \prime}}{a} \simeq \frac{1}{\eta^{2}}(2+3(\epsilon-\tau)) .
$$

By introducing $\widetilde{v}:=(\epsilon-\tau)+3 / 2$ and keeping only up to first orders of the first and second slow roll parameters (10) could be rewritten as

$$
\chi^{\prime \prime}+\left(k^{2}-\frac{\widetilde{v}^{2}-1 / 4}{\eta^{2}}\right) \chi=0
$$

where $\widetilde{\nu}^{2} \simeq 3(\epsilon-\tau)+9 / 4$. Solving the above Bessel like equation, the magnitude of $\phi$ can be achieved as

$$
|\phi| \simeq \frac{H(1-\epsilon)}{k}\left(\frac{k}{a H(1-\epsilon)}\right)^{3 / 2-\widetilde{v}} .
$$

To estimate the best value of $\epsilon$, one can use the power spectrum concept for instance in light of Planck 2013 [1]. It is obvious that, by considering the Fourier transformation for an arbitrary function as

$$
\widetilde{\Phi}_{\mathbf{k}}(t, \mathbf{r})=\int \frac{d^{3} k}{(2 \pi)^{3} \sqrt{2 k}} e^{j \mathbf{k} \cdot \mathbf{r}} \widetilde{\phi}_{\mathbf{k}}(t),
$$

where $\mathbf{k}$ is comoving momentum and $\mathbf{r}$ is spatial vector, the power spectrum can be defined as

$$
\left\langle\widetilde{\phi}_{\mathbf{k}}(t) \widetilde{\phi}_{\mathbf{k}^{\prime}}^{*}(t)\right\rangle=\frac{2 \pi^{2}}{k^{2}} \rho_{S}(2 \pi)^{3} \delta^{3}\left(\mathbf{k}-\mathbf{k}^{\prime}\right),
$$


where $\rho_{S}$ is power spectrum in question and $\left\langle\widetilde{\phi}_{\mathbf{k}}(t) \widetilde{\phi}_{\mathbf{k}^{\prime}}^{*}(t)\right\rangle$ indicates the mean square value of $\widetilde{\phi}_{\mathbf{k}}(t)$. By combining (7), (14), and (15) the power spectrum can be achieved as

$$
\rho_{S}=\frac{k^{2}}{(2 \pi)^{2}}|\phi(t)|^{2} .
$$

To achieve this result the relation between annihilation and creation operators reads $\left[a_{\mathbf{k}}, a_{\mathbf{k}^{\prime}}^{\dagger}\right]=(2 \pi)^{3} \delta^{3}\left(\mathbf{k}-\mathbf{k}^{\prime}\right)$. At last if one considers the relation (13), the power spectrum could be rewritten as

$$
\rho_{S}=(1-2 \epsilon) H^{2}\left(\frac{k}{a H(1-\epsilon)}\right)^{3-2 \widetilde{v}} .
$$

Also another important quantity is notable that is the spectral index which plays a crucial role in inflationary investigations and is defined as

$$
n_{S}-1=\frac{d \ln \rho_{S}}{d \ln k} .
$$

Substituting (17) into the above equation, one has

$$
n_{S}-1=(1-2 \epsilon)(3-2 \widetilde{\nu}),
$$

and at last after some manipulations the spectral index is obtained as $n_{S}-1=-2 \epsilon+2 \tau$. By means of observed value of spectral index, $n_{S}<0.9675$, risen from Planck 2013 [1], the best value of the first slow roll parameter is $\epsilon \simeq 0.02$.

2.1. Typical Example: Massless Scalar Field. In this case we want to consider massless scalar field. Therefore if, in action (1), one assumes $V(\Phi)=0$, solving (9), for positive modes, is attained as

$$
\phi(\eta)=\frac{1}{a(\eta)}\left(1-\frac{i \xi}{k \eta}\right) e^{(-i k \eta)}
$$

where $\xi=(2+3 \epsilon) / 2$. In this case, as massive ones, the first slow roll parameter is considered only up to the first order. For this typical case we want to estimate the zero point quantum fluctuation contribution in the energy budget of the universe. To begin we have to calculate the vacuum expectation value of the scalar field energy-momentum tensor. By means of (5) and (20), one has

$$
\begin{aligned}
\rho_{\mathrm{vac}} & =\left\langle 0\left|T_{00}^{\phi}\right| 0\right\rangle \\
& =\frac{1}{2} \int_{0}^{a(t) \Lambda_{c}} \frac{d^{3} k}{(2 \pi)^{3} 2 k}\left[|\dot{\phi}|^{2}+\frac{k^{2}}{a^{2}(t)}|\phi|^{2}\right], \\
P_{\mathrm{vac}} & =\frac{1}{3} \Sigma_{i}\left\langle 0\left|T_{i}^{\phi i}\right| 0\right\rangle \\
& =\frac{1}{2} \int_{0}^{a(t) \Lambda_{c}} \frac{d^{3} k}{(2 \pi)^{3} 2 k}\left[|\dot{\phi}|^{2}-\frac{k^{2}}{3 a^{2}(t)}|\phi|^{2}\right],
\end{aligned}
$$

where $\rho_{\mathrm{vac}}$ and $P_{\mathrm{vac}}$, indicate the energy density and pressure of the vacuum quantum fluctuations, respectively. According to quantum field theory the cutoff $\Lambda_{c}$ should be considered greater than physical momenta $k / a(t)$. By considering (20) and definition of scale factor for a de-Sitter like background, one has

$$
\begin{aligned}
\left(|\dot{\phi}|^{2}+\frac{k^{2}}{a^{2}(t)}|\phi|^{2}\right)= & \frac{1}{a^{4}}\left[2 k^{2}+a^{2} H^{2}(1+9 \epsilon)\right] \\
& +\mathcal{O}\left(\epsilon^{2}\right), \\
\left(|\dot{\phi}|^{2}-\frac{k^{2}}{3 a^{2}(t)}|\phi|^{2}\right)= & \frac{2}{3 a^{4}}\left[k^{2}-\frac{a^{2} H^{2}}{2}(1-3 \epsilon)\right] \\
& +\mathcal{O}\left(\epsilon^{2}\right) .
\end{aligned}
$$

By virtue of (22), solving (21) leads to

$$
\begin{aligned}
& \rho_{\mathrm{vac}}=\frac{\Lambda_{c}^{4}}{16 \pi^{2}}+(1+9 \epsilon) \frac{H^{2}(t) \Lambda_{c}^{2}}{16 \pi^{2}}, \\
& P_{\mathrm{vac}}=\frac{\Lambda_{c}^{4}}{48 \pi^{2}}-(1-3 \epsilon) \frac{H^{2}(t) \Lambda_{c}^{2}}{48 \pi^{2}} .
\end{aligned}
$$

The first terms in (23) are the contribution of the energy density and pressure for Minkowskian space time; and because of the cutoff dependency the latter terms are well known bare quantities. To get rid of quartic divergencies the subtraction mechanism is a good suggestion, which is close to Casimir approach [9]. The base of the Casimir effect is on the subtraction mechanism. In this effect the energy that gives rise to observable effects is the difference between vacuum energy risen from two parallel conducting plates, for example, for electromagnetic field and vacuum energy computed in Minkowskian space-time in an infinite volume. Therefore when two infinite values for energy of space time and plates are subtracted one can obtain a finite quantity. Therefore by means of Arnowitt-Deser-Misner (ADM) approach [2] and subtraction mechanism one concludes that the vacuum energy to an asymptotically flat space-time with metric $g_{\mu \nu}$ can be achieved as $E=H_{\mathrm{GR}}\left(g_{\mu \nu}\right)-H_{\mathrm{GR}}\left(\eta_{\mu \nu}\right)$, where $H_{\mathrm{GR}}$ refers to the Hamiltonian which is calculated in general relativity. This equation indicates that flat space-time does not gravitate and the contribution of the energy which is obtained in Minkowski space-time can be subtracted from related quantity in curved background [2, 9-11]. Thence, because flat space-time does not gravitate one is able to subtract the contribution of quartic terms in Minkowski space from the same terms in FriedmannLemaitreRobertsonWalker (FLRW) space-time. Also it should be stressed that the results which were obtained for de-Sitter like scenario have some notable differences with normal de-Sitter ones. The first is, for deSitter like investigations, the Hubble parameter is not a constant and this causes the appearance of the first slow roll parameter in the model which could be considered to investigate the accuracy of this model. As a second note, the coefficients which appeared in energy density and pressure cause the increase of the zero point quantum fluctuations 
contribution in the dark energy. Now let us investigate the bare quantities which are

$$
\begin{aligned}
& \rho_{\text {bare }}=(1+9 \epsilon) \frac{H^{2}(t) \Lambda_{c}^{2}}{16 \pi^{2}}, \\
& P_{\text {bare }}=-(1-3 \epsilon) \frac{H^{2}(t) \Lambda_{c}^{2}}{48 \pi^{2}} .
\end{aligned}
$$

Following [3] one can introduce counter terms for energy density and pressure, respectively, as follows:

$$
\begin{aligned}
& \rho_{\text {count }}=-(1+9 \epsilon) \frac{H^{2}(t) \Lambda_{c}^{2}}{16 \pi^{2}}+\rho_{Z}, \\
& P_{\text {count }}=(1-3 \epsilon) \frac{H^{2}(t) \Lambda_{c}^{2}}{48 \pi^{2}}+P_{Z},
\end{aligned}
$$

where

$$
\begin{aligned}
& \rho_{Z}=(1+9 \epsilon) \frac{H^{2}(t) M^{2}}{16 \pi^{2}}, \\
& P_{Z}=-(1-3 \epsilon) \frac{H^{2}(t) M^{2}}{48 \pi^{2}},
\end{aligned}
$$

where subscript $Z$ refers to the zero point and $M$ is in order of Planck mass. The interesting result which could be realized from (27) and (28) is that they appear to contain a mix of ultraviolet (UV) cutoff, $M$ as Planck mass, and infrared (IR) cutoff, and $H(t)$ as the inverse of horizon size. Based on quantum field theory concepts, we know the widely separated energy scales are decoupled; but one can find that the origin of this mix of UV-IR cutoffs arises from classical subtraction procedure, that is, $E=H_{G R}\left(g_{\mu \nu}\right)-H_{G R}\left(\eta_{\mu \nu}\right)$. In fact the origin of this mix goes back to (21), where it involves $T_{\mu \nu}^{\phi}$ and $\left\langle 0\left|T_{\mu \nu}^{\phi}\right| 0\right\rangle$ as local and global quantities, respectively. For more discussion we refer the reader to $[2,11,12]$. Using (27) and (28), the equation of state (EoS) parameter for the vacuum fluctuations could be expressed as

$$
\omega_{Z}=\frac{P_{Z}}{\rho_{Z}}=\frac{-1}{3}+4 \epsilon .
$$

This relation indicates that the EoS of zero point quantum fluctuations is dependent on the first slow roll parameter. It should be noted also that whereas this approach is similar to Casimir mechanism both positive and negative signs for energy density are acceptable. To consider this fact one can consider coefficient $\sigma= \pm 1$, for (27), and redefines it as

$$
\rho_{Z}=\sigma(1+9 \epsilon) \frac{H^{2}(t) M^{2}}{16 \pi^{2}} .
$$

The positive sign causes an attractive force and negative ones are related to the repulsive case. Now for more discussions about time dependency of energy density of vacuum fluctuations, one is able to redefine it based on critical energy density of the universe. Hence considering definition of critical energy density $\left(\rho_{\text {cri }}=3 H^{2}(t) / 8 \pi G\right)$ and by means of definition of Planck mass $M_{\mathrm{Pl}}=1 / \sqrt{G}$, ( $G$ is the Newtonian constant), the energy density of zero point fluctuations could be rewritten as

$$
\rho_{Z}=\Omega_{Z} \rho_{\text {cri }}+\mathcal{O}\left(\epsilon^{2}\right)=\beta \frac{1+9 \epsilon}{\epsilon^{2}} a^{-2 \epsilon}(t),
$$

where $\Omega_{Z}=\beta(1+9 \epsilon) / M_{\mathrm{Pl}}^{2}$ and $\beta=\sigma M^{2} / 16 \pi^{2}$. Because of the time dependency of $\rho_{Z}(t)$, the conservation equation for $\rho_{Z}$ is not satisfied. Hence using $\dot{\rho}_{Z}=-2 \epsilon H(t) \rho_{Z}$ and (31) one has

$$
\dot{\rho}_{Z}+3 H(t) \rho_{Z}\left(1+\omega_{Z}\right)=Q,
$$

where $Q$ is dissipation function and it could be obtained as

$$
Q=2 H(t) \rho_{Z}(1+5 \epsilon) .
$$

Therefore the energy density of quantum fluctuations is capable of exchanging energy with other components of the universe. To investigate the transformation of energy we consider some different cases as follows.

\section{Transformation of Energy between Different Components of the Universe}

3.1. Transformation of Energy between Zero Point Fluctuations and Matter. In this case one has

$$
\begin{aligned}
\dot{\rho}_{Z}+3 H(t) \rho_{Z}\left(1+\omega_{Z}\right) & =2 H(t) \rho_{Z}(1+5 \epsilon), \\
\dot{\rho}_{m}+3 H(t) \rho_{m} & =-2 H(t) \rho_{Z}(1+5 \epsilon),
\end{aligned}
$$

and therefore the combination of the two sections of the above equation yields

$$
\dot{\rho}_{Z}+3 H(t) \rho_{Z}\left(1+\omega_{Z}\right)+\dot{\rho}_{m}+3 H(t) \rho_{m}=0,
$$

which indicates that the conservation equation in general is satisfied. Therefore by means of (31) and (35), $\rho_{m}$ could be achieved as

$$
\rho_{m}=\Psi a^{-2 \epsilon}(t)+\widetilde{\Psi} a^{-3}(t),
$$

where $\Psi=-2 \beta(1+44 \epsilon / 3) / 3 \epsilon^{2}$ and $\widetilde{\Psi}$ is integration constant. From (36) it is realized that in our model the matter density equation is modified, where the first term indicates the matters which are risen from interaction of quantum fluctuations with matter and the latter indicates the remaining contribution of matter, namely, ordinary cold dark matter.

3.2. Transformation of Energy between Zero Point Energy and the Remnant Components of Dark Energy. Assume there is an internal interaction between different components of dark energy, namely, $\rho_{\Lambda}$ and $\rho_{Z}$, where $\rho_{\Lambda}$ indicates energy density of cosmological constant. In this case, one can suppose that $\omega_{\Lambda}=-1$ and therefore the conservation equation reads

$$
\dot{\rho}_{Z}+3 H(t) \rho_{Z}\left(1+\omega_{Z}\right)+\dot{\rho}_{\Lambda}=0 .
$$


By virtue of $\dot{\rho}_{Z}=-2 \beta(1+3 \epsilon) /\left(\epsilon^{2} a^{3 \epsilon}(t)\right)$ and definition of scale factor in de-Sitter like background, one has

$$
\rho_{\Lambda}=\frac{\beta}{\epsilon}(1+14 \epsilon) H^{2}(t)+C_{0}
$$

where $C_{0}$ is integration constant. In addition it is obvious that because $\dot{\rho}_{Z}$ is proportional to $\dot{\rho}_{\Lambda}$ the Big Bang Nucleosynthesis (BBN) constraint which has been discussed in [2] could be considered to estimate the upper bound on $\Omega_{Z}=$ $\beta(1+9 \epsilon) / M_{\mathrm{Pl}}^{2}$. Also it should be stressed that, by comparing $\rho_{Z}$ with the one in normal de-Sitter model, it is clear that the coefficient $(1+9 \epsilon)$ causes the increase of the magnitude of zero point energy density.

3.3. Transformation of Energy between All Components of the Universe. In this stage, one can consider a general case where all components of the universe are in an interaction. Therefore the conservation equations could be written as

$$
\begin{array}{r}
\dot{\rho}_{m}+3 H(t) \rho_{m}=\widetilde{Q}_{i} \\
\dot{\rho}_{\Lambda}+\dot{\rho}_{Z}+3 H(t) \rho_{Z}\left(1+\omega_{Z}\right)=-\widetilde{Q}_{i},
\end{array}
$$

where $\widetilde{Q}_{i}$ are dissipation functions and are defined as follows:

(i) $\widetilde{Q}_{1}=3 \kappa H(t) \rho_{\Lambda}$,

(ii) $\widetilde{Q}_{2}=3 \gamma H(t) \rho_{m}$,

(iii) $\widetilde{Q}_{3}=3 \theta H(t) \rho_{Z}$.

A also $\kappa, \gamma$, and $\theta$ indicate the strength of the interaction between different components of the universe [13].

3.3.1. Solving Conservation Equation for $\widetilde{Q}_{1}$. By virtue of (40) and dissipation function $\widetilde{Q}_{1}$, the conservation equation for dark energy components of the universe is as follows:

$$
\dot{\rho}_{\Lambda}+\dot{\rho}_{Z}+3 \kappa H(t) \rho_{\Lambda}+3 H(t) \rho_{Z}\left(1+\omega_{Z}\right)=0 .
$$

Based on (31) and (29), (41) could be rewritten as

$$
\dot{\rho}_{\Lambda}+3 \kappa H(t) \rho_{\Lambda}+2 \beta(1+14 \epsilon) H^{3}(t)=0,
$$

and hence solving this differential equation yields

$$
\rho_{\Lambda}=\frac{-\breve{D}}{(3 \kappa-2 \epsilon)} a^{-2 \epsilon}(t)+\widetilde{c} a^{-3 \kappa},
$$

where $\breve{D}=2(1+14 \epsilon) \beta / \epsilon^{2}$ and $\widetilde{c}$ is integration constant. By substituting (43) into (39) one can attain $\rho_{m}$ as follows

$$
\begin{aligned}
\rho_{m}= & \frac{-\breve{D}}{(3-2 \epsilon(1-1 / \kappa))} a^{-2 \epsilon}(t)+\frac{\kappa \widetilde{c}}{1-\kappa} a^{-3 \kappa} \\
& +\breve{B} a^{-3}(t) .
\end{aligned}
$$

In the above equation $\breve{B}$ is integration constant. From this relation one can conclude that, in matter equation, only ordinary cold dark matter does not appear; rather, an extra term appears which is risen from interaction of matter and quantum fluctuations, namely, subdark matter. In the following, we will come back to this issue.
3.3.2. Solving Conservation Equation for $\widetilde{Q}_{2}$. By rewriting (39) and (40) for $\widetilde{Q}_{2}$, one arrives to

$$
\begin{aligned}
\dot{\rho}_{m}+3 H(t) \rho_{m} & =3 \gamma H(t) \rho_{m}, \\
\dot{\rho}_{\Lambda}+\dot{\rho}_{Z}+3 H(t) \rho_{Z}\left(1+\omega_{Z}\right) & =-3 \gamma H(t) \rho_{m},
\end{aligned}
$$

and thus (45) could be considered as

$$
\dot{\rho}_{m}+3 H(t)(1-\gamma) \rho_{m}=0,
$$

and after solving, one can attain

$$
\rho_{m}=\rho_{m 0} a^{-3(1-\gamma)}(t) .
$$

Substituting (48) into (46) yields

$$
\rho_{\Lambda}=\frac{\beta(1+14 \epsilon)}{\epsilon} H^{2}(t)+\frac{\rho_{m 0} \gamma}{3(1-\gamma)} a^{-3(1-\gamma)}+\mathfrak{M},
$$

where $\mathfrak{M}$ is the integration constant.

3.3.3. Solving Conservation Equation for $\widetilde{Q}_{3}$. In this stage, one can suppose that the interaction between different components of the universe is determined by virtue of $\widetilde{Q}_{3}$. Thence (39) and (40) are rearranged as

$$
\begin{aligned}
\dot{\rho}_{m}+3 H(t) \rho_{m} & =3 \theta H(t) \rho_{Z} \\
\dot{\rho}_{\Lambda}+\dot{\rho}_{Z}+3 H(t) \rho_{Z}\left(1+\omega_{Z}\right) & =-3 \theta H(t) \rho_{Z} .
\end{aligned}
$$

Using definition of scale factor in de-Sitter like background, one is allowed to rewrite (50) as

$$
\dot{\rho}_{m}+\frac{3}{\epsilon} \rho_{m} a^{-\epsilon}-\frac{3 \theta}{\epsilon}\left(\frac{\beta(1+3 \epsilon)}{\epsilon^{2}}\right) a^{-3 \epsilon}=0,
$$

and solving this equation for $\rho_{m}$ yields

$$
\rho_{m}=\breve{A} a^{-2 \epsilon}+\breve{K} a^{-3}(t)
$$

where $\breve{A} \simeq 3 \theta \beta(1+8 \epsilon) / \epsilon^{2}$ and $\breve{K}$ is the integration constant. Hence, to attain $\rho_{\Lambda}$, from (51) one has

$$
\dot{\rho}_{\Lambda}+\frac{\beta}{\epsilon^{3}}[2+3 \theta+27 \epsilon(1+\theta)] a^{-3 \epsilon}(t)=0,
$$

and solution of this equation for $\rho_{\Lambda}$ reads

$$
\rho_{\Lambda}=\frac{3 \beta}{\epsilon}[2+3 \theta+27 \epsilon(1+\theta)] H^{2}(t) \text {. }
$$

\section{Bounds Which Are Risen from Time Evolution of Dark Energy}

In this section we want to compare the results of this work with results which are risen from standard $\Lambda$ CDM model. For this end, one can start from the Friedmann equation. Therefore, the ratio of dark energy density and critical energy 
density in the standard model as a function of red shift parameter, $z$, is obtained as

$$
\frac{\rho_{\mathrm{DE}}(t)}{\rho_{\mathrm{cri}}(t)}=\frac{\Omega_{\Lambda}(1+z)^{3\left(1+\omega_{\Lambda}\right)}}{\Omega_{M}(1+z)^{3}+\Omega_{\Lambda}} .
$$

It should be emphasized in the above equation that one can get $\Omega_{\Lambda}=0.73, \Omega_{M}=0.23$, and $\omega_{\Lambda}=-0.98$, which are obtained from a combination of CMB, BAO, and SNeIa data sets [2,14-16]. Whereas, in this work, the components of dark energy are quantum fluctuations and cosmological constant, thence the Friedmann equation is obtained as

$$
\begin{aligned}
\frac{3 H^{2}(t)}{8 \pi G} & =\left(\rho_{m}(t)+\rho_{\mathrm{DE}}\right) \\
& =\left(\rho_{m}(t)+\rho_{\Lambda}(t)+\Omega_{Z} \rho_{\text {cri }}(t)\right),
\end{aligned}
$$

where subscript DE indicates dark energy. By substituting (31) into (57), the Friedmann equation could be rewritten as

$$
H^{2}(t)=\frac{H_{0}^{2}(t)}{1-\Omega_{z}}\left(\Omega_{m}(t)+\Omega_{\Lambda}(t)\right),
$$

where $\Omega_{i}(t)=\rho_{i}(t) / \rho_{\text {cri }}(0)$, and $i$ refers to $m, \Lambda$, and $Z$, respectively; in addition $\rho_{\text {cri }}(0)$ denotes the critical energy density in present epoch. It should be noted that the energy densities of curvature and radiation are neglected. From (31) and definition of dimensionless energy density parameters, one gets

$$
\rho_{\mathrm{DE}}=\Omega_{Z} \rho_{\mathrm{cri}}(t)+\rho_{\mathrm{cri}}(0) \Omega_{\Lambda} .
$$

By virtue of definition of $\rho_{\text {cri }}(t),(58)$ could be rearranged as

$$
\rho_{\text {cri }}(t)=\frac{\rho_{\text {cri }}(0)}{1-\Omega_{Z}}\left[\Omega_{m}(t)+\Omega_{\Lambda}\right] .
$$

By dividing $\rho_{\mathrm{DE}}$ and $\rho_{\mathrm{cri}}(t)$, one arrives to

$$
\frac{\rho_{\mathrm{DE}}(t)}{\rho_{\text {cri }}(t)}=\Omega_{Z}+\frac{\left(1-\Omega_{Z}\right) \Omega_{\Lambda}}{\Omega_{m}(t)+\Omega_{\Lambda}} .
$$

Whereas different equations for $\rho_{m}$ (based on different conditions for interaction) are attained, $\Omega_{m}(t)$ in (60) gets different forms. Thus by considering (36), (44), (53), and (61) it could be rewritten, respectively, as follows.

(a) From (36), consider

$$
\begin{aligned}
& \frac{\rho_{\mathrm{DE}}(t)}{\rho_{\mathrm{cri}}(t)} \\
& \quad=\Omega_{Z} \\
& \quad+\frac{\left(1-\Omega_{Z}\right)\left(\Omega_{\mathrm{DE}}-\Omega_{Z}\right)}{\Omega_{m}(1+z)^{3}+\widetilde{\Omega}_{m}(1+z)^{2 \epsilon}+\left(\Omega_{\mathrm{DE}}-\Omega_{Z}\right)},
\end{aligned}
$$

where $\widetilde{\Omega}_{m}=\Psi / \rho_{\text {cri }}(0)$ and $\Omega_{m}=\widetilde{\Psi} / \rho_{\text {cri }}(0)$. In Figure 1, the evolution of (62) versus $z$ parameter shows a deviation in comparison to the ordinary $\rho_{\mathrm{DE}}(t) / \rho_{\text {cri }}(t),(56)$ that illuminates the effects of $\Omega_{Z}$ and $\epsilon$ in the evolution of this function. (b) From (44), consider

$\frac{\rho_{\mathrm{DE}}(t)}{\rho_{\mathrm{cri}}(t)}=\Omega_{Z}$
$\quad+\frac{\left(1-\Omega_{Z}\right)\left(\Omega_{\mathrm{DE}}-\Omega_{Z}\right)}{\Omega_{m}(1+z)^{3}+\bar{\Omega}_{m}(1+z)^{2 \epsilon}+\Omega_{m}^{*}(1+z)^{3 \kappa}+\left(\Omega_{\mathrm{DE}}-\Omega_{Z}\right)}$,

where $\bar{\Omega}_{m}=-\breve{D} / \rho_{\text {cri }}(0)(3-2 \epsilon(1-1 / \kappa))$ and $\Omega_{m}^{*}=$ $\kappa \widetilde{c} / \rho_{\text {cri }}(0)(1-\kappa)$. Considering the above equation, it is found out that the $\widetilde{Q}_{1}$ case does not lead to physical result, because the equation for $\rho_{m}$ can not satisfy observations as well.

(c) From (48), consider

$$
\frac{\rho_{\mathrm{DE}}(t)}{\rho_{\mathrm{cri}}(t)}=\Omega_{Z}+\frac{\left(1-\Omega_{Z}\right)\left(\Omega_{\mathrm{DE}}-\Omega_{Z}\right)}{\Omega_{m}(1+z)^{3(1-\gamma)}+\left(\Omega_{\mathrm{DE}}-\Omega_{Z}\right)},
$$

where $\Omega_{m}(1+z)^{3(1-\gamma)}$ indicates dark matter component of the universe. Figure 2 shows a deviation with respect to ordinary $\rho_{\mathrm{DE}}(t) / \rho_{\mathrm{cri}}(t)(56)$ where in comparison to Figure 1 it is realized that $Q_{2}$ function has better results.

(d) From (53), consider

$$
\begin{aligned}
& \frac{\rho_{\mathrm{DE}}(t)}{\rho_{\mathrm{cri}}(t)} \\
& =\Omega_{Z} \\
& \quad+\frac{\left(1-\Omega_{Z}\right)\left(\Omega_{\mathrm{DE}}-\Omega_{Z}\right)}{\Omega_{m}(1+z)^{3}+\Omega_{m}^{\sharp}(1+z)^{2 \epsilon}+\left(\Omega_{\mathrm{DE}}-\Omega_{Z}\right)},
\end{aligned}
$$

where $\Omega_{m}=\breve{K} / \rho_{\text {cri }}(0)$ and $\Omega_{m}^{\sharp}=\breve{A} / \rho_{\text {cri }}(0)$. The energy density parameter in this case behaves as the case related to (38). This manner is similar to case (a) that is (62), and therefore it does not need to plot its evolution. It should be noted that the behaviour of $\rho_{\mathrm{DE}}(z) / \rho_{\text {cri }}(z)$ based on different quantities for $\Omega_{Z}$ is plotted in Figure 3.

As it is obvious, Figure 3 illuminates the evolution of $\rho_{\mathrm{DE}}(z) / \rho_{\text {cri }}(z)$ versus $z$ (62) for three different values $\Omega_{Z}=$ $0.16,0.05$, and -0.16 . The results are shown with green dashed, red dotted, and blue solid lines, respectively. It is clear that $\rho_{\mathrm{DE}}(z) / \rho_{\text {cri }}(z)$ decreases with decreasing $\Omega_{Z}$.

\section{Conclusion and Discussion}

Vacuum quantum fluctuations in a de-Sitter like background for both massive and massless bosonic scalar fields have been investigated. In light of Planck database 2013 we have estimated the best value for the first slow roll parameter and using such quantity the different components of the Universe's energy budget have been calculated. It should be stressed that the scalar fields have been quantized in a FLRW framework and it has been shown that the contribution of vacuum fluctuations has increased in such background in comparison 


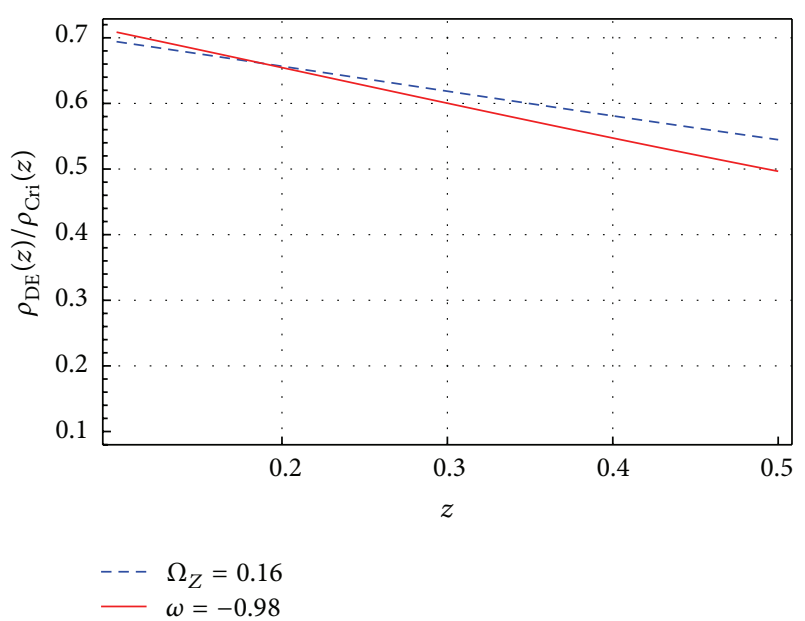

FIGURE 1: $\rho_{\mathrm{DE}}(z) / \rho_{\text {cri }}(z)$ versus $z$ have been shown with dashedblue line (62) and solid-red line, respectively (56). The auxiliary parameters are $\Omega_{Z}=0.16, \Omega_{m}=0.17, \widetilde{\Omega}_{m}=0.1, \Omega_{\mathrm{DE}}=0.73$, $\epsilon=0.02$, and $\omega_{\Lambda}=-0.98$.

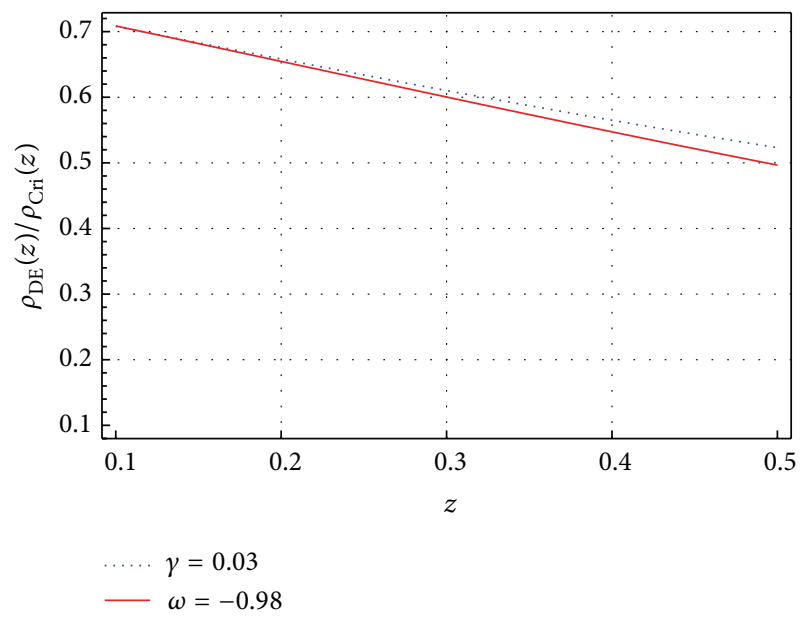

FIGURE 2: $\rho_{\text {DE }}(z) / \rho_{\text {cri }}(z)$ versus $z$ have been shown with dotted line (64) and solid line (56). The auxiliary parameters are $\Omega_{Z}=0.16$, $\Omega_{m}=0.23, \Omega_{\mathrm{DE}}=0.73, \gamma=0.03$, and $\omega_{\Lambda}=-0.98$.

with normal de-Sitter case. It should be emphasized that the subtraction approach has been used to eliminate the infinities which appeared in the calculations. Incidentally, using the physical energy density of zero point quantum fluctuation, it has been realized that this component of the universe has to have an interaction with other components of the universe. In addition, when the energy density of matter is achieved, it has been found that beside ordinary dark matter there exist matter components which were created due to interaction with zero point quantum fluctuations. Also whereas zero point energy density was time dependent, the transformation of energy between different ingredients of the universe has been investigated. It was considerable that, for the state in which all components of the universe exchange energy between themselves, time evolution of $\rho_{\mathrm{DE}}(z) / \rho_{\text {cri }}(z)$ has shown that $Q_{2}=3 \gamma H \rho_{m}$ is in best agreement in comparison

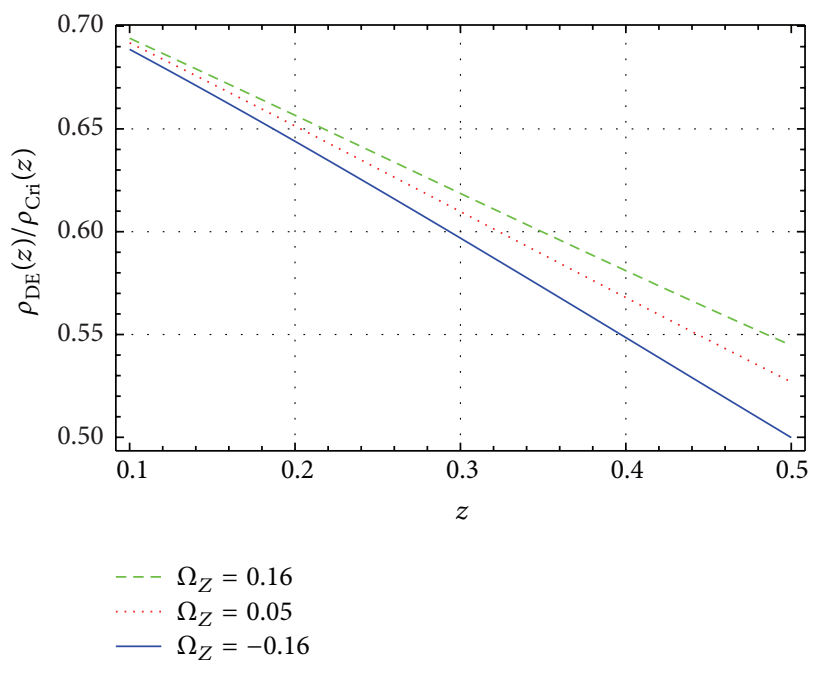

FIGURE 3: $\rho_{\text {DE }}(z) / \rho_{\text {cri }}(z)$ versus $z$ (62) for three different values $\Omega_{Z}=$ $0.16, \Omega_{Z}=0.05$, and $\Omega_{Z}=-0.16$. They have been shown with dashed-green, dotted-red, and blue (solid) lines, respectively.

with observational database and also the interaction term $\widetilde{Q}_{1}=3 \kappa H(t) \rho_{\Lambda}$ had not any physical results. At last for more details, the bounds which have risen from time evolution of dark energy density in comparison to standard $\Lambda$ cosmology have been investigated. To compare the results of this work with observational data, we have regarded the time evolution of $\rho_{\mathrm{DE}}(z) / \rho_{\text {cri }}(z)$ which was concluded from a combination of CMB, BAO, and SNeIa data sets. From Figures 2 and 3, the evolution of (62) and (64) versus $z$ in comparison to observational results has been illustrated.

\section{Competing Interests}

The authors declare that there is no conflict of interests regarding the publication of this paper. Haidar Sheikhahmadi declares that the received funding mentioned in the "Acknowledgments" section did not lead to any conflict of interests regarding the publication of this paper.

\section{Acknowledgments}

Haidar Sheikhahmadi would like to thank Iran's National Elites Foundation for financial support during this work. He expresses his appreciation to Professor Y. Sobouti for sharing their pearls of wisdom with him during the course of this research.

\section{References}

[1] A. Abergel, P. A. R. Ade, N. Aghanim et al., "Planck 2013 results. XI. All-sky model of thermal dust emission," Astronomy \& Astrophysics, vol. 571, p. A11, 2014.

[2] M. Maggiore, "Zero-point quantum fluctuations and dark energy," Physical Review D, vol. 83, no. 6, Article ID 063514, 16 pages, 2011. 
[3] L. Hollenstein, M. Jaccard, M. Maggiore, and E. Mitsou, "Zeropoint quantum fluctuations in cosmology," Physical Review D, vol. 85, no. 12, Article ID 124031, 23 pages, 2012.

[4] M. Bordag, G. L. Klimchitskaya, U. Mohideen, and V. M. Mostepanenko, Advances in the Casimir Effect, Oxford University Press, Oxford, UK, 2009.

[5] L. E. Parker and D. J. Toms, Quantum Field Theory in Curved Spacetime: Quantized Field and Gravity, Cambridge Monographs on Mathematical Physics, Cambridge University Press, Cambridge, UK, 2009.

[6] N. D. Birell and P. C. W. Davies, Quantum Field Theory in Curved Spacetime, Cambridge Univesity Press, 1982.

[7] A. R. Liddle and D. H. Lyth, Cosmological Inflation and Largescale Structure, Cambridge University Press, Cambridge, Uk, 2000.

[8] D. Langlois, "Inflation, quantum fluctuations and cosmological perturbations," in Particle Physics and Cosmology: The Interface, pp. 235-278, Springer Netherlands, 2005.

[9] H. B. G. Casimir, "On the attraction between two perfectly conducting plates," Proceedings of the Koninklijke Nederlandse Akademie van Wetenschappen B, vol. 51, pp. 793-795, 1948.

[10] L. Parker and S. A. Fulling, "Adiabatic regularization of the energy-momentum tensor of a quantized field in homogeneous spaces," Physical Review D, vol. 9, no. 2, pp. 341-354, 1974.

[11] T. Padmanabhan, "Vacuum fluctuations of energy density can lead to the observed cosmological constant," Classical and Quantum Gravity, vol. 22, no. 17, pp. L107-L112, 2005.

[12] E. Kh. Akhmedov, "Vacuum energy and relativistic invariance," http://arxiv.org/abs/hep-th/0204048.

[13] R. G. Cai, Z. L. Tuo, H. B. Zhang, and Q. Su, "Notes on ghost dark energy," Physical Review D, vol. 84, no. 12, Article ID 123501, 2011.

[14] A. G. Riess, A. V. Filippenko, P. Challis et al., "Observational evidence from supernovae for an accelerating universe and a cosmological constant," The Astronomical Journal, vol. 116, no. 3, article 1009, 1998.

[15] H. V. Peiris, E. Komatsu, L. Verde et al., "First-year Wilkinson Microwave Anisotropy Probe (WMAP) WMAP is the result of a partnership between Princeton University and NASA's Goddard Space Flight Center. Scientific guidance is provided by the WMAP Science Team. Observations: Implications For Inflation," The Astrophysical Journal Supplement Series, vol. 148, no. 1, p. 213, 2003.

[16] E. Komatsu, K. M. Smith, J. Dunkley et al., "Seven-year wilkinson microwave anisotropy probe $\left(\mathrm{WMAP}^{*}\right)$ observations: cosmological interpretation," The Astrophysical Journal Supplement Series, vol. 192, no. 2, article 18, 2011. 

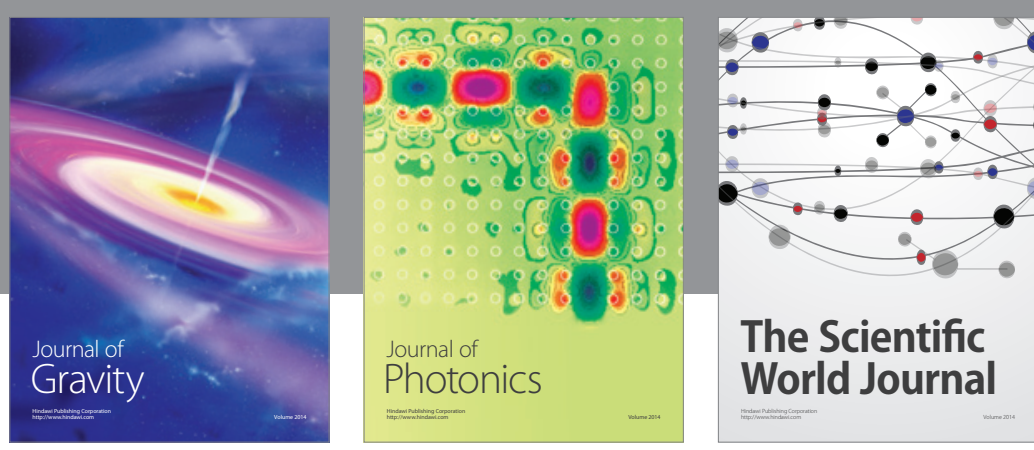

The Scientific World Journal
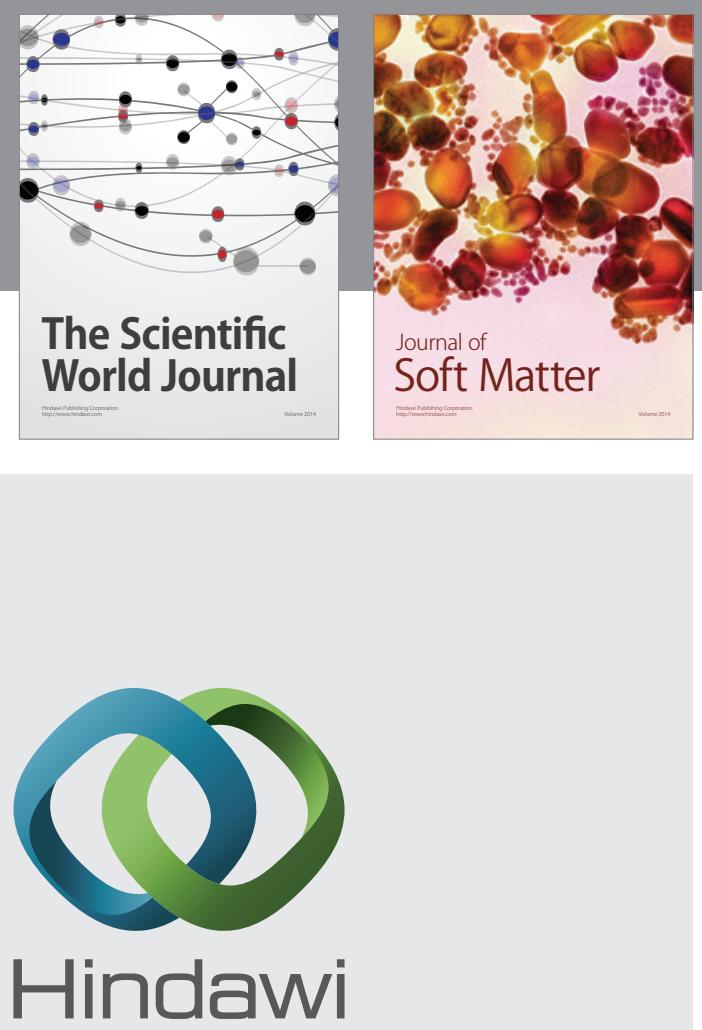

Submit your manuscripts at

http://www.hindawi.com

nternational Journal of

Statistical Mechanics
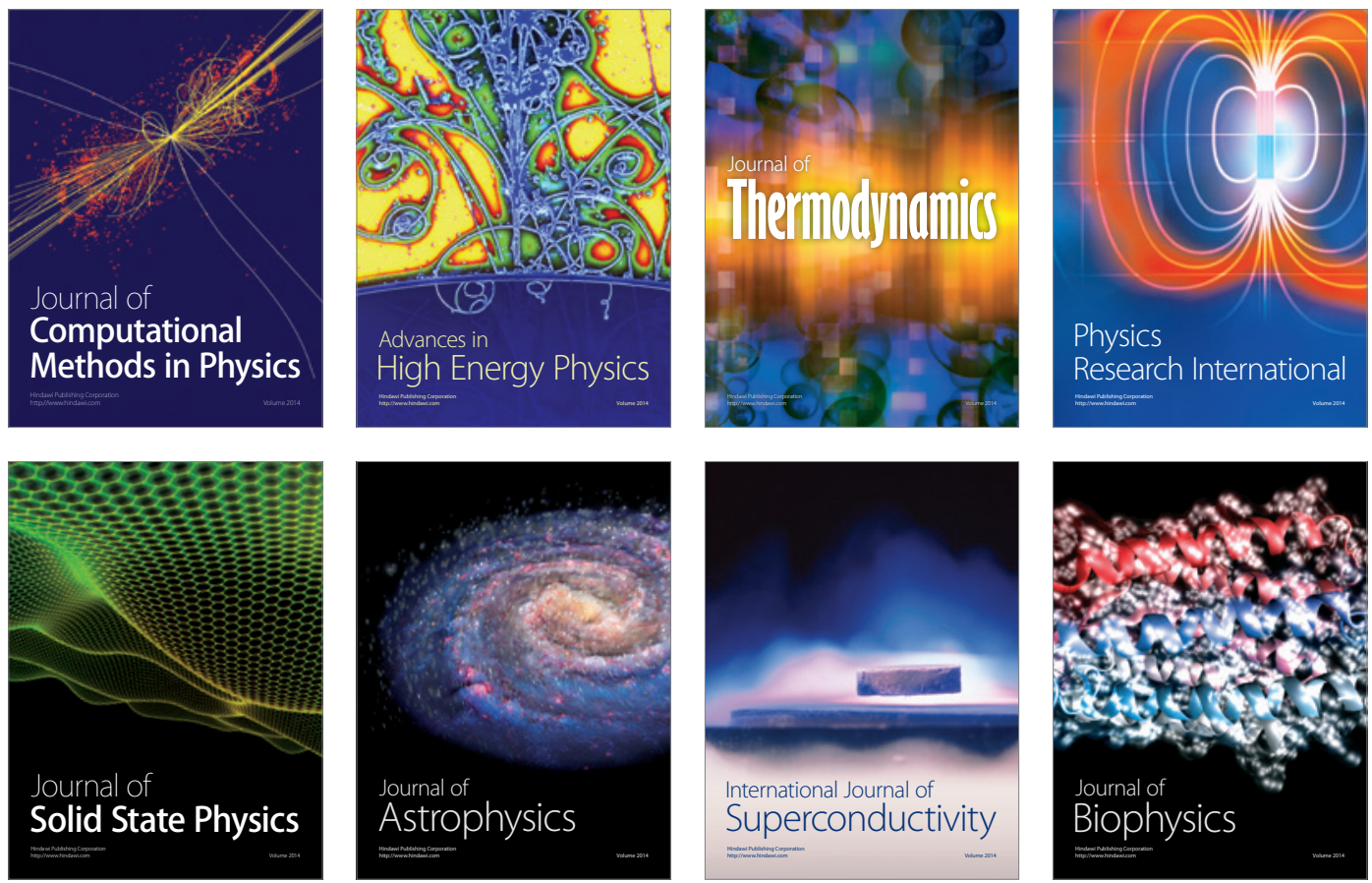
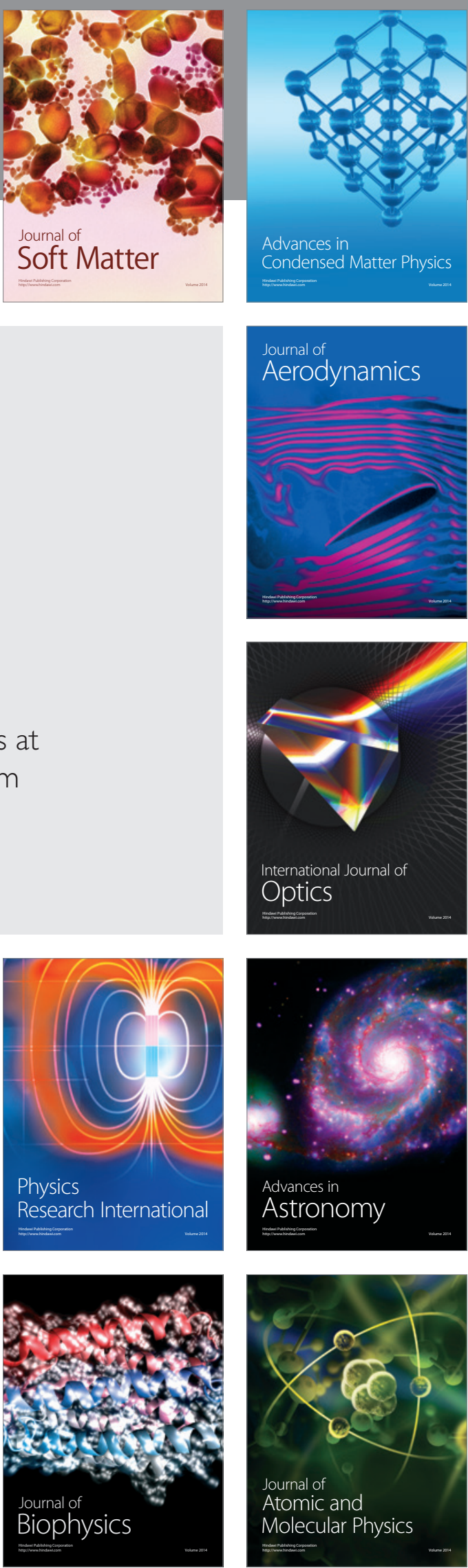